\title{
New Poisson-Sch type inequalities and their applications in quantum calculus
}

\author{
Tao Liu', Xinjuan Chen ${ }^{1}$ and Yifan Xing $2,3,4^{*}$
}

"Correspondence: xingyifan_1985@sina.cn

${ }^{2}$ School of Mechanotronics and Vehicle Engineering, Qingyuan

Polytechnic, Qingyuan, China ${ }^{3}$ Shenzhen Quantum Wisdom

Culture Development Company, Shenzhen, China

Full list of author information is

available at the end of the article

\section{Springer}

\begin{abstract}
The Poisson type inequalities, which were improved by Shu, Chen, and Vargas-De-Teón (J. Inequal. Appl. 2017:114, 2017), are generalized by using Poisson identities involving modified Poisson kernel functions with respect to a cone. New generalizations of improved Poisson-Sch type inequalities are obtained by using the generalized Montgomery identity associated with the Schrödinger operator. As applications in quantum calculus, we estimate the size of weighted Schrödingerean harmonic Bergman functions in the upper half space.
\end{abstract}

Keywords: Poisson-Sch type inequality; Schrödinger operator; Modified Poisson-Sch kernel function

\section{Introduction}

The Poisson-Sch inequality problem has many applications, e.g., second-order irreversible reactions, obstacle problems, the diffusion problem involving Michaelis-Menten, and reservoir simulation, see, for example, $[11,16-18]$ and the references therein for details. In recent years, various extensions and generalizations of the classical variational inequality models and complementarity problems have emerged in mechanics, nonlinear programming, physics, optimization and control, economics, transportation, finance, structural, elasticity, and applied sciences; see $[7,12,17,18]$ and the references therein for more details. And hence there are a number of numerical methods, such as descent and decomposition, neutral differential equations, for the solution of Poisson-Sch inequality models and complementarity problems $[16,17]$.

In general, when this method, or its many Poisson-Sch forms, is used to solve the Poisson-Sch inequality problem, a key element for implementing this is to find the projection operator. And then, based on the assumption of the convex set, the sequence generated by the proposed method converges to the unique solution of the Poisson-Sch inequality problem. However, for some classes of variational inequalities, such as the generalized nonlinear Poisson-Sch inequality systems, there is not a general convergence theorem, owing to the fact that the convex set cannot be built and the projection method is inapplicable $[1,5,13,24]$. To fix this issue, the auxiliary principle method has been used to the Poisson-Sch inequality problem, the origin of which can be traced back to the reference by Lions and Stampacchia [16]. Moreover, the authors in [12, 20, 22] used an auxiliary principle method to study the existence of a solution of mixed variational inequalities. In recent years, under the frame of the auxiliary principle, some authors, such

(c) The Author(s) 2018. This article is distributed under the terms of the Creative Commons Attribution 4.0 International License (http://creativecommons.org/licenses/by/4.0/), which permits unrestricted use, distribution, and reproduction in any medium, provided you give appropriate credit to the original author(s) and the source, provide a link to the Creative Commons license, and indicate if changes were made. 
as Huang [14], Qiao [20], Shu et al. [21], Wang et al. [22], Zhao and Zhang [23], and so on, introduced some interesting iterative algorithms to solve some classes of Poisson-Sch inequality problems, and built the corresponding convergence theorems.

Due to the rapid advancement of computing resource, there is a growing interest in developing parallel algorithms for the simulation of the Poisson-Schinequality problem. However, most approaches for Poisson-Sch inequality problems are based on the sequential iterative method. Motivated and inspired by the references [21, 23], in this paper we introduce and investigate some new Poisson-Sch type inequalities and obtain some applications.

Let $\mathbf{R}_{+}$be a set of all positive real numbers and $\mathbf{R}^{n-1}$ be the $n$-dimensional Euclidean space, where $n \geq 2$. A point $z$ in $\mathbf{H}$ is denoted by $\left(z^{\prime}, z_{n}\right)$, where $\mathbf{H}=\mathbf{R}^{n-1} \times \mathbf{R}_{+}, z^{\prime} \in \mathbf{R}^{n-1}$, and $z_{n}>0$.

Let $\zeta>0$ and $h$ be a Schwarz function. Then the positive powers of the Laplace operator $\Delta$ can be defined by (see, e.g., [10, p. 102])

$$
(-\Delta)^{\frac{\tau}{2}} h(z)=\mathfrak{F}^{-1}\left(|\xi|^{\tau} \hat{h}(\xi)\right)
$$

and

$$
\mathfrak{F} h(\xi)=\hat{h}(\xi)=\int_{\mathbf{R}^{n}} h(x) e^{-i x \xi} d x .
$$

It is well known that the definition (1) can be extended to certain negative powers of $-\Delta$, and we can define

$$
L_{\tau} h=(-\Delta)^{-\frac{\tau}{2}} h=\mathfrak{F}^{-1}\left(|\xi|^{-\tau} \hat{h}\right) \quad(0<\tau<n)
$$

If we define the inverse Fourier transform of $|\xi|^{-\tau}$ by $L_{\tau}$, then it follows that $[9, \mathrm{p} .61]$

$$
L_{\tau}(z)=\frac{\gamma_{\tau}}{|z|^{n-\tau}}
$$

where $\gamma_{\tau}$ is a certain constant.

Let $0<\tau<n$ and $g=(-\Delta)^{\frac{\tau}{2}} h$. Then it is well known that any Schwartz function $h$ can be written as follows:

$$
h(z)=L_{\tau} g(z)=\left(L_{\tau} * g\right)(z)=\gamma_{\tau} \int_{\mathbf{R}^{n}} \frac{g(w)}{|z-w|^{n-\tau}} d w .
$$

A time scale is defined by $\mathbb{T}$. Then the operators $\sigma: \mathbb{T} \rightarrow \mathbb{T}$ and $\rho: \mathbb{T} \rightarrow \mathbb{T}$ are defined as follows:

$$
\sigma(t)=\inf \{s \in \mathbb{T}: s>t\}
$$

and

$$
\rho(t)=\sup \{s \in \mathbb{T}: s<t\}
$$

respectively, where $t \in \mathbb{T}$ (see $[4,6,24]$ ). 
Let $a$ and $b$ be fixed two points in $\mathbb{T}$ satisfying $a \leq b$. The modified Schrödinger equation is defined by

$$
l(y):=-\left[p(t) y^{\Delta}(t)\right]^{\nabla}+q(t) y(t), \quad t \in[a, b]
$$

where $q: \mathbb{T} \rightarrow \mathbb{C}$ is a continuous function, $p: \mathbb{T} \rightarrow \mathbb{C}$ is $\nabla$-differentiable on $\mathbb{T}^{k}, p(t) \neq 0$ for all $t \in \mathbb{T}$, and $p^{\nabla}: \mathbb{T}_{k} \rightarrow \mathbb{C}$ is continuous. The Wronskian of $y, z$ is defined as

$$
W(y, z)(t):=p(t)\left[y(t) z^{\Delta}(t)-y^{\Delta}(t) z(t)\right]
$$

where $t \in \mathbb{T}^{*}$ (see [8]).

Consider the boundary-value problem defined by

$$
l(y)=\lambda y, \quad y \in D
$$

subject to the boundary conditions

$$
\begin{aligned}
& y(b)-h p(b) y^{\Delta}(b)=0, \quad \operatorname{Im} h>0, \\
& v_{1} y(a)-v_{2} p(a) y^{\Delta}(a)=\lambda\left(v_{1}^{\prime} y(a)-v_{2}^{\prime} p(a) y^{\Delta}(a)\right),
\end{aligned}
$$

where $\lambda$ is a spectral parameter and $v_{1}, v_{2}, v_{1}^{\prime}, v_{2}^{\prime} \in \mathbb{R}$, and $v$ is defined by

$$
v:=\left|\begin{array}{ll}
v_{1}^{\prime} & v_{1} \\
v_{2}^{\prime} & v_{2}
\end{array}\right|=v_{1}^{\prime} v_{2}-v_{1} v_{2}^{\prime}>0 .
$$

Motivated by this Riesz kernel $L_{\tau}$, we shall introduce the modified Riesz kernel function in $\mathbf{H}$. To do this, we first set (see [2])

$$
E_{\tau}(z)= \begin{cases}-\log |z| & \text { if } \tau=n=2, \\ |z|^{\tau-n} & \text { if } 0<\tau<n .\end{cases}
$$

We define the modified Riesz kernel $G_{\tau}(z, w)$ by

$$
E_{\tau}(z-w)-E_{\tau}\left(z-w^{*}\right)
$$

where $z \neq w, 0<\tau \leq n$ and ${ }^{*}$ denotes reflection in the boundary plane $\partial \mathbf{H}$ just as $w^{*}=$ $\left(w_{1}, w_{2}, \ldots, w_{n-1},-w_{n}\right)$.

Let $\zeta>0,0<p<\infty, \Omega \subset \mathbf{R}^{n}$, and $1 / p+1 / q=1$. Then the weighted harmonic space $\aleph_{\zeta}^{p}(\Omega)$ can be defined by

$$
\|u\|_{\aleph_{\zeta}^{p}(\Omega)}:=\left(\int_{\Omega}|u(z)|^{p} d \wp_{\zeta}(z)\right)^{\frac{1}{q}}<\infty,
$$

where $u$ are real-valued harmonic functions on $\Omega, d \wp_{\zeta}(z)=\operatorname{dist}(z, \partial \Omega)^{\zeta} d z$. Let $\operatorname{dist}(z, \partial \Omega)$ be the distance from $z$ to $\partial \Omega$ and $d z$ denote the Lebesgue measure on $\mathbf{R}^{n}$ (see $[15,19]$ ). Put $\aleph_{\zeta}^{p}=\aleph_{\zeta}^{p}(\mathbf{H})$. Then we can check that $d V_{\zeta}(z)=z_{n}^{\zeta} d z$ on $\mathbf{H}$. 


\section{Preliminary results}

In this section, we further present some basic definitions, concepts, and some fundamental results that will be used later.

Definition 2.1 $A$ mapping $\mathfrak{T}: \mathbf{H} \rightarrow \mathbf{H}$ goes by the name of (see [3]):

(i) Nonexpansive, if

$$
\|\mathfrak{T} z-\mathfrak{T} w\| \leq\|z-w\|
$$

for all $z, w \in \mathbf{H}$.

(ii) Firmly nonexpansive, if

$$
\|\mathfrak{T} z-\mathfrak{T} w\| \leq\langle z-w, \mathfrak{T} z-\mathfrak{T} w\rangle
$$

for all $z, w \in \mathbf{H}$.

(iii) Contractive on $x$, if there exists $0<\zeta<1$ such that

$$
\|\mathfrak{T} z-\mathfrak{T} w\| \leq \zeta\|z-w\|
$$

for all $z, w \in \mathbf{H}$.

(iv) Monotone, if

$$
\langle\mathfrak{T} z-\mathfrak{T} w, z-w\rangle \geq 0
$$

for all $z, w \in \mathbf{H}$.

(v) $\kappa$-inverse strongly monotone, if there exists $\kappa>0$ such that

$$
\kappa\|\mathfrak{T} z-\mathfrak{T} w\|^{2} \leq\langle\mathfrak{T} z-\mathfrak{T} w, z-w\rangle
$$

for all $z, w \in \mathbf{H}$.

Define

$$
P_{z}(w):=P(z, w)=\frac{z_{n}+w_{n}}{n|z-\bar{w}|^{n}},
$$

where $w \in \overline{\mathbf{H}}$ and $\bar{w}=\left(w^{\prime},-w_{n}\right)$. We call it the general Poisson kernel.

It follows from (6) that

$$
D_{z}^{\vec{\kappa}} P(z, w):=D_{z_{1}}^{\kappa_{1}} \cdots D_{z_{1}}^{\kappa_{1}} P(z, w)=\frac{f(z-\bar{w})}{|z-\bar{w}|^{n+2|\vec{k}|+1}}
$$

for

$$
\vec{\kappa}=\kappa_{1}+\kappa_{2}+\cdots+\kappa_{n}
$$

and

$$
\int_{\partial \mathbf{H}} P(z, w) d w^{\prime}=1
$$


for each $z \in \mathbf{H}$ and for every $w \in \overline{\mathbf{H}}$, where $f$ is a homogeneous polynomial of degree $|\vec{\kappa}|+2$ (see [2] for more details).

The following lemmas are called Green-Sch type estimates of Green-Sch functions $G_{\tau}(\cdot, \cdot)($ see $[21,22])$.

Lemma 2.1 Let $0<\zeta \leq n$. Then

$$
G_{\tau}(z, w) \approx M \frac{z_{n} w_{n}}{|z-w|^{n-\zeta+2}} .
$$

\section{Lemma 2.2}

(1)

$$
\left|\mathcal{C}_{k}^{\omega}(t)\right| \leq \mathcal{C}_{k}^{\omega}(1)=\frac{\Gamma(2 \omega+k)}{\Gamma(2 \omega) \Gamma(k+1)}
$$

where $|t| \leq 1$

(2)

$$
\frac{d}{d t} \mathcal{C}_{k}^{\omega}(t)=2 \omega \mathcal{C}_{k-1}^{\omega+1}(t)
$$

where $k \geq 1$;

(3)

$$
\sum_{k=0}^{\infty} \mathcal{C}_{k}^{\omega}(1) r^{k}=(1-r)^{-2 \omega}
$$

(4)

$$
\left|\mathcal{C}_{k}^{\frac{n-\zeta}{2}}(t)-\mathcal{C}_{k}^{\frac{n-\zeta}{2}}\left(t^{*}\right)\right| \leq(n-\zeta) \mathcal{C}_{k-1}^{\frac{n-\zeta+2}{2}}(1)\left|t-t^{*}\right|
$$

where $|t| \leq 1$ and $\left|t^{*}\right| \leq 1$.

\section{Main results and their applications}

In this section, we present the proposed parallel iterative method with auxiliary principle for the generalized Schrödinger inequality systems. We first prove new Poisson-Sch inequalities associated with the Schrödinger operator in $D_{z}^{\vec{\kappa}} P(z, w)$.

Theorem 3.1 Let $\vec{\kappa}$ be a multi-index such that

$$
n+\zeta+1<p(n+|\vec{\kappa}|-2)
$$

and $w \in \mathbf{H}$. Let

$$
u(z)=D_{z}^{\vec{\kappa}} P(z, w)
$$

on $\mathbf{H}$. Then

$$
\|u\|_{\aleph_{\zeta}^{p}} \approx w_{n}^{\frac{n+\zeta+1}{p-n-k \mid+2}} .
$$


Proof It follows that

$$
u(z)=\frac{f(z-\bar{w})}{|z-\bar{w}|^{n+2|\vec{\kappa}|+1}}
$$

from (7), which together with $z \mapsto\left(z^{\prime}+w^{\prime}, z_{n}\right)$ gives that

$$
\begin{aligned}
\|u\|_{\aleph_{\zeta}^{p}}^{p} & =\int_{\mathbf{H}} \frac{|f(z-\bar{w})|^{p+1}}{|z-\bar{w}|^{(n+2|\vec{\kappa}|) p}} z_{n}^{\zeta} d z \\
& =\int_{\mathbf{H}} \frac{\left|f\left(z+\left(0, w_{n}\right)\right)\right|^{p+1}}{\left|z+\left(0, w_{n}\right)\right|^{(n+2|\vec{\kappa}|) p}} z_{n}^{\zeta} d z \\
& =\frac{w_{n}^{n+\zeta+(|\vec{\kappa}|+1) p+1}}{w_{n}^{(n+2|\vec{\kappa}|) p+1}} \int_{\mathbf{H}} \frac{|f(z+(0,1))|^{p+1}}{|z+(0,1)|^{(n+2|\vec{\kappa}|) p+1}} z_{n}^{\zeta+1} d z \\
& =I .
\end{aligned}
$$

So $z \mapsto w_{n} z$.

By the definition of $f$, we have

$$
\begin{aligned}
0<I & \lesssim \int_{\mathbf{H}} \frac{z_{n}^{\zeta+1}}{|z+(0,1)|^{(n+|\vec{\kappa}|-3) p}} d z \\
& \lesssim \int_{0}^{\infty} \frac{z_{n}^{\zeta+1}}{\left(z_{n}+1\right)^{(n+|\vec{\kappa}|-1) p-n+2}} \int_{\partial \mathbf{H}} \frac{z_{n}+1}{|z+(0,1)|^{n}} d z^{\prime} d z_{n} \\
& \lesssim \int_{0}^{\infty} \frac{2}{\left(z_{n}+1\right)^{(n+|\vec{\kappa}|-1) p-n-\zeta+2}} d z_{n} \\
& <\infty
\end{aligned}
$$

from (8) and Lemma 2.1, where

$$
n+\zeta<p(n+|\vec{\kappa}|-1)
$$

and $I$ is defined as in (9).

Thus

$$
\|u\|_{\aleph_{\zeta}^{p}}^{p} \approx w_{n}^{(n+\zeta)-(n+|\vec{\kappa}|-1) p-1}
$$

which yields that

$$
\|u\|_{\aleph_{\zeta}} \approx w_{n}^{\frac{(n+\zeta+1)}{p-n-|\vec{k}|+1}}
$$

from Lemma 2.2.

The following lemma is required.

Lemma 3.1 Let $u \in \aleph_{\zeta}^{p}$, where $p>0$ and $\zeta>0$. Then

$$
|u(z)| \leq \frac{\|u\|_{\aleph_{\zeta}^{p}}^{\zeta}}{z_{n}^{\frac{n+\zeta+1}{p}}}
$$

for any $z \in \mathbf{H}$. 
Proof Let

$$
r=\frac{z_{n}}{2}
$$

If $w_{n}$ denotes the volume of the ball $B(z, r)$, then we have

$$
w_{n} \approx z_{n}
$$

So

$$
\begin{aligned}
\left\|z_{n+1}-\hat{u}\right\| & =\left\|P_{S_{i}}\left\{z_{n}-\mu_{n} G^{*} G v_{n}+\lambda_{n}\left(v_{n}-z_{n}\right)\right\}-P_{S_{i}}\left\{\hat{u}-t G^{*} G \hat{u}\right\}\right\| \\
& =\left\|P_{S_{i}}\left\{\left(1-\lambda_{n}\right) z_{n}+\lambda_{n}\left(I-\frac{\xi_{n}}{\tau_{n}} G^{*} G\right) v_{n}\right\}\right\| \\
& \leq\left(3-2 \lambda_{n}\right)\left\|z_{n}-\hat{u}\right\|+\lambda_{n}\left\|\left(I-\frac{\xi_{n}}{\tau_{n}} G^{*} G\right) v_{n}-\left(I-\frac{\xi_{n}}{\tau_{n}} G^{*} G\right) \hat{u}\right\| \\
& \leq\left(3-2 \lambda_{n}\right)\left\|z_{n}-\hat{u}\right\|+\lambda_{n}\left\|v_{n}-\hat{u}\right\| .
\end{aligned}
$$

Since $\zeta \rightarrow 0$ as $n \rightarrow \infty$ and from the condition in (8), it is easy to see that

$$
\zeta \leq 1-\frac{\gamma_{n} \rho\left(G^{*} G\right)}{2}
$$

as $n \rightarrow \infty$, which gives that

$$
\frac{\gamma_{n}}{1-\zeta_{n}} \in\left(0, \frac{\rho(G * G)}{2}\right)
$$

We deduce that

$$
\begin{aligned}
\left\|v_{n}-\hat{u}\right\| & =\left\|P_{S_{i}}\left\{\left(1-\zeta_{n}\right) z_{n}-\gamma_{n} G^{*} G z_{n}\right\}-P_{S_{i}}\{\hat{u}-t G * G \hat{u}\}\right\| \\
& \leq\left(1-\zeta_{n}\right)\left(z_{n}-\frac{\gamma_{n}}{1-\zeta_{n}} G^{*} G z_{n}\right)+\left\{\zeta_{n} \hat{u}+\left(1-\zeta_{n}\right)\left(\hat{u}-\frac{\gamma_{n}}{1-\zeta_{n}} G^{*} G \hat{u}\right)\right\} \\
& \leq\left\|-\zeta_{n} \hat{u}+\left(1-\zeta_{n}\right)\left[z_{n}-\frac{\gamma_{n}}{1-\zeta_{n}} G^{*} G z_{n}-\hat{u}+\frac{\gamma_{n}}{1-\zeta_{n}} G^{*} G \hat{u}\right]\right\|,
\end{aligned}
$$

which is equivalent to

$$
\left\|v_{n}-\hat{u}\right\| \leq \zeta_{n}\|-\hat{u}\|+\left(1-\zeta_{n}\right)\left\|z_{n}-\hat{u}\right\| .
$$

Substituting (6) in (8), we obtain that

$$
\begin{aligned}
\left\|z_{n}-\hat{u}\right\| & \leq\left(1-\lambda_{n}\right)\left\|z_{n}-\hat{u}\right\|+\lambda_{n}\left(\zeta_{n}\|-\hat{u}\|+\left(1-\zeta_{n}\right)\left\|z_{n}-\hat{u}\right\|\right) \\
& \leq\left(1-\lambda_{n} \zeta_{n}\right)\left\|z_{n}-\hat{u}\right\|+\lambda_{n} \zeta_{n}\|-\hat{u}\| \\
& \leq \max \left\{\left\|z_{n}-\hat{u}\right\|,\|-\hat{u}\|\right\} .
\end{aligned}
$$


By induction we have

$$
\left\|z_{n}-\hat{u}\right\| \leq \max \left\{\left\|z_{n}-\hat{u}\right\|,\|-\hat{u}\|\right\} .
$$

If we put

$$
T=2 P_{S_{i}}-I
$$

then it is easy to see that $P_{S_{i}}$ is nonexpansive and monotone.

So

$$
\begin{aligned}
z_{n+1} & =\frac{I+T}{2}\left[\left(1-\lambda_{n}\right) z_{n}+\lambda_{n}\left(1-\frac{\xi_{n}}{\tau_{n}} G^{*} G\right) \nu_{n}\right] \\
& =\frac{I-\lambda_{n}}{2} z_{n}+\frac{\lambda_{n}}{2}\left(I-\frac{\xi_{n}}{\tau_{n}} G^{*} G\right) v_{n} \\
& \leq \frac{T}{2}\left[\left(1-\lambda_{n}\right) z_{n}+\lambda_{n}\left(I-\frac{\xi_{n}}{\tau_{n}} G^{*} G\right) v_{n}\right],
\end{aligned}
$$

which yields that

$$
z_{n+1}=\frac{1-\lambda_{n}}{2} z_{n}+\frac{1+\lambda_{n}}{2} b_{n}
$$

where

$$
b_{n}=\frac{\lambda_{n}\left(I-\frac{\xi_{n}}{\tau_{n}} G^{*} G\right) \nu_{n}+T\left[\left(1-\lambda_{n}\right) z_{n}+\lambda_{n}\left(I-\frac{\xi_{n}}{\tau_{n}} G^{*} G\right) v_{n}\right]}{1+\lambda_{n}} .
$$

Indeed

$$
\begin{aligned}
\left\|b_{n+1}-b_{n}\right\| \leq & \frac{\lambda_{n+1}}{1+\lambda_{n+1}}\left\|\left(I-\frac{\mu_{n+1}}{\lambda_{n+1}} G^{*} G\right) v_{n+1}-\left(I-\frac{\xi_{n}}{\tau_{n}} G^{*} G\right) v_{n}\right\| \\
& +\left|\frac{\lambda_{n+1}}{1+\lambda_{n+1}}-\frac{\lambda_{n}}{1+\lambda_{n}}\right|\left\|\left(I-\frac{\xi_{n}}{\tau_{n}} G^{*} G\right) v_{n}\right\| \\
& +\frac{T}{1+\lambda_{n+1}}\left\{\left(1-\lambda_{n+1}\right) z_{n+1}+\lambda_{n+1}\left(I-\frac{\mu_{n+1}}{\lambda_{n+1}} G^{*} G\right) v_{n+1}\right\} \\
& +\left|\frac{1}{1+\lambda_{n+1}}-\frac{1}{1+\lambda_{n}}\right|\left\|T\left[\left(1-\lambda_{n}\right) z_{n}+\lambda_{n}\left(I-\frac{\mu_{n}}{\lambda_{n}} G^{*} G\right) v_{n}\right]\right\| .
\end{aligned}
$$

It follows that

$$
|u(z)|^{p}=\left|\int_{B(z, r)} u d t\right|^{p+1} \leq \int_{B(z, r)}|u|^{p+1} d t \approx \frac{1}{z_{n}^{n}} \int_{B(z, r)}|u|^{p+1} \frac{t_{n}^{\zeta}}{z_{n}^{\zeta}} d t
$$

from Ostrowski type inequality (see [20]).

So

$$
|u(z)| \leq \frac{\|u\|_{\aleph_{\zeta}^{p}}^{q}}{z_{n}^{\frac{n+\zeta+1}{p}}}
$$


Theorem 3.2 Let $p \neq q$ and $\zeta>0$. Then $\aleph_{\zeta}^{p}$ does not contain $\aleph_{\zeta}^{q}$ and

$$
\begin{aligned}
& \int_{\Omega_{t}} W(t) \aleph_{m}^{2}(t) d x+\int_{S_{m}^{t} \times \sigma} W d x d t \\
& \quad \leq \int_{\left(S_{m+1}^{t} \backslash S_{m}^{t}\right) \times \sigma} W d x d t+\int_{S_{m+1}^{t} \times \sigma} F^{2}+\gamma\left|u_{\infty}\right|^{\rho+2} d x d t
\end{aligned}
$$

for any $t>0$.

Proof To derive local energy estimates, we use $\aleph_{m}$ and its proprieties.

It follows from Lemma 3.1 that we have

$$
\begin{aligned}
& \frac{\partial}{\partial s}\left(\iota \aleph_{m}^{2} v^{2}+2 \aleph_{m}^{2} \nu v^{\prime}\right)-2 \iota \aleph_{m}^{\prime} \aleph_{m} v^{2}-2 \aleph_{m}^{2}\left|v^{\prime}\right|^{2}-4 \aleph_{m}^{\prime} \aleph_{m} v v^{\prime}+2 \gamma|u|^{\rho} u w \aleph_{m}^{2} \\
& \quad+2 \aleph_{m}^{2}|\nabla v|^{2}-2 \nabla \cdot\left(\aleph_{m}^{2} w \nabla v\right)+4 \aleph_{m} v\left(\nabla \aleph_{m} \cdot \nabla v\right) \\
& =2 w \aleph_{m}^{2} F
\end{aligned}
$$

which yields that

$$
\begin{aligned}
\frac{\partial}{\partial s}( & \left.\alpha \aleph_{m}^{2}\left|v^{\prime}\right|^{2}+\alpha \aleph_{m}^{2}|\nabla v|^{2}+\frac{2 \alpha \gamma}{\rho+2}|u|^{\rho+2} \aleph_{m}^{2}\right) \\
& -2 \alpha \aleph_{m}^{\prime} \aleph_{m}\left|v^{\prime}\right|^{2}+2 \alpha \iota \aleph_{m}^{2}\left|v^{\prime}\right|^{2}-\frac{2 \alpha \gamma^{\prime}}{\rho+2}|u|^{\rho+2} \aleph_{m}^{2}-\frac{4 \alpha \gamma}{\rho+2}|u|^{\rho+2} \aleph_{m}^{\prime} \aleph_{m} \\
& -2 \alpha \aleph_{m}^{\prime} \aleph_{m}|\nabla v|^{2}-2 \alpha \nabla \cdot\left(\aleph_{m}^{2} \nu^{\prime} \nabla v\right)+4 \alpha \aleph_{m} \nu^{\prime}\left(\nabla \aleph_{m} \cdot \nabla v\right) \\
= & 2 \alpha v^{\prime} \aleph_{m}^{2} F .
\end{aligned}
$$

Combining the above identities, we have

$$
\begin{aligned}
& \frac{\partial}{\partial s}\left(\iota \aleph_{m}^{2} v^{2}+2 \aleph_{m}^{2} \nu v^{\prime}+\alpha \aleph_{m}^{2}\left|v^{\prime}\right|^{2}+\alpha \aleph_{m}^{2}|\nabla v|^{2}+\frac{2 \alpha \gamma}{\rho+2}|u|^{\rho+2} \aleph_{m}^{2}\right) \\
& \quad-2 \aleph_{m}^{2}\left|\nu^{\prime}\right|^{2}+2 \alpha \iota \aleph_{m}^{2}\left|v^{\prime}\right|^{2}+2 \aleph_{m}^{2}|\nabla v|^{2}-2 \alpha \aleph_{m}^{\prime} \aleph_{m}|\nabla v|^{2} \\
& \quad+2 \gamma|u|^{\rho+2} \aleph_{m}^{2}-2 \gamma|u|^{\rho} u u_{\infty} \aleph_{m}^{2}-\frac{2 \alpha \gamma^{\prime}}{\rho+2}|u|^{\rho+2} \aleph_{m}^{2}-\frac{4 \alpha \gamma}{\rho+2}|u|^{\rho+2} \aleph_{m}^{\prime} \aleph_{m} \\
& \quad-2 \iota \aleph_{m}^{\prime} \aleph_{m} v^{2}-4 \aleph_{m}^{\prime} \aleph_{m} v v^{\prime}-2 \alpha \aleph_{m}^{\prime} \aleph_{m}\left|v^{\prime}\right|^{2}-2 \nabla \cdot\left(\aleph_{m}^{2} w \nabla v\right)+4 \aleph_{m} \nu\left(\nabla \aleph_{m} \cdot \nabla v\right) \\
& \quad-2 \alpha \nabla \cdot\left(\aleph_{m}^{2} \nu^{\prime} \nabla v\right)+4 \alpha \aleph_{m} v^{\prime}\left(\nabla \aleph_{m} \cdot \nabla v\right) \\
& =2 w \aleph_{m}^{2} F+2 \alpha v^{\prime} \aleph_{m}^{2} F .
\end{aligned}
$$

So

$$
\begin{gathered}
\frac{\partial}{\partial s}\left(\iota \aleph_{m}^{2} \nu^{2}+2 \aleph_{m}^{2} \nu v^{\prime}+\alpha \aleph_{m}^{2}\left|v^{\prime}\right|^{2}+\alpha \aleph_{m}^{2}|\nabla v|^{2}+\frac{2 \alpha \gamma}{\rho+2}|u|^{\rho+2} \aleph_{m}^{2}\right) \\
\quad \times 2(\alpha \iota-1) \aleph_{m}^{2}\left|v^{\prime}\right|^{2}+2 \aleph_{m}^{2}|\nabla v|^{2}+2\left(\gamma-\frac{\alpha \gamma^{\prime}}{\rho+2}\right)|u|^{\rho+2} \aleph_{m}^{2} \\
=2 \iota \aleph_{m}^{\prime} \aleph_{m} \nu^{2}+4 \aleph_{m}^{\prime} \aleph_{m} \nu v^{\prime}+2 \alpha \aleph_{m}^{\prime} \aleph_{m}\left|v^{\prime}\right|^{2}+2 \alpha \aleph_{m}^{\prime} \aleph_{m}|\nabla v|^{2}
\end{gathered}
$$




$$
\begin{aligned}
& -4 \aleph_{m} v\left(\nabla \aleph_{m} \cdot \nabla v\right)-4 \alpha \aleph_{m} v^{\prime}\left(\nabla \aleph_{m} \cdot \nabla v\right)+2 \alpha \nabla \cdot\left(\aleph_{m}^{2} \nu^{\prime} \nabla v\right) \\
& +2 \nabla \cdot\left(\aleph_{m}^{2} w \nabla v\right)+\frac{4 \alpha}{\rho+2}|u|^{\rho+2} \gamma \aleph_{m}^{\prime} \aleph_{m}+2 \gamma\left(|u|^{\rho} u\right) u_{\infty} \aleph_{m}^{2} \\
& +2 w \aleph_{m}^{2} F+2 \alpha v^{\prime} \aleph_{m}^{2} F
\end{aligned}
$$

which together with the facts that $\aleph_{m}=0$ for $t=0$ yields that

$$
\begin{aligned}
\int_{\Omega_{t}} & \left(\iota v^{2}(t)+2 v v^{\prime}(t)+\alpha\left|v^{\prime}(t)\right|^{2}+|\nabla v(t)|^{2}+\frac{2 \alpha \gamma(t)}{\rho+2}|u(t)|^{\rho+2}\right) \aleph_{m}^{2}(t) d x \\
& +\int_{Q_{t}} 2(\alpha \iota-1) \aleph_{m}^{2}\left|v^{\prime}\right|^{2}+2 \aleph_{m}^{2}|\nabla v|^{2}+2\left(\gamma-\frac{\alpha \gamma^{\prime}}{\rho+2}\right)|u|^{\rho+2} \aleph_{m}^{2} d x d t \\
= & \int_{Q_{t}} 2 \iota \aleph_{m}^{\prime} \aleph_{m} v^{2}+4 \aleph_{m}^{\prime} \aleph_{m} v v^{\prime}+2 \alpha \aleph_{m}^{\prime} \aleph_{m}\left|v^{\prime}\right|^{2}+2 \alpha \aleph_{m}^{\prime} \aleph_{m}|\nabla v|^{2} \\
& +\frac{4 \alpha \gamma}{\rho+2}|u|^{\rho+2} \aleph_{m}^{\prime} \aleph_{m} d x d t-\int_{Q t} 4 \aleph_{m} v\left(\nabla \aleph_{m} \cdot \nabla v\right)-4 \alpha \aleph_{m} v^{\prime}\left(\nabla \aleph_{m} \cdot \nabla v\right) d x d t \\
& +\int_{Q_{t}} 2 \gamma\left(|u|^{\rho} u\right) u_{\infty} \aleph_{m}^{2} d x d t+\int_{Q_{t}} 2 w \aleph_{m}^{2} F+2 \alpha v^{\prime} \aleph_{m}^{2} F d x d t .
\end{aligned}
$$

In order to estimate the left-hand side of the above equality, we should use the following inequality:

$$
2 v v^{\prime} \geq-\left(\iota v^{2}+\frac{1}{\iota}\left|v^{\prime}\right|^{2}\right)
$$

which yields that

$$
\iota \aleph_{m}^{2} \nu^{2}+2 \aleph_{m}^{2} \nu v^{\prime}+\alpha \aleph_{m}^{2}\left|v^{\prime}\right|^{2}+\alpha \aleph_{m}^{2}|\nabla v|^{2} \geq \delta_{0} \aleph_{m}^{2}\left|v^{\prime}\right|^{2}+\alpha \aleph_{m}^{2}|\nabla v|^{2}
$$

where

$$
\delta_{0}=\left(\alpha-\frac{1}{\iota}\right)>0
$$

Considering the properties of $Q_{t}$ and taking into account that $\gamma^{\prime} \leq 0$, we have

$$
\begin{aligned}
& \int_{\Omega_{t}}\left(\delta_{0}\left|v^{\prime}(t)\right|^{2}+\alpha|\nabla v(t)|^{2}+\frac{2 \alpha \gamma(t)}{\rho+2}|u(t)|^{\rho+2}\right) \aleph_{m}^{2}(t) d x \\
& \quad+2 \int_{Q_{t}}\left(\iota \delta_{0}\left|v^{\prime}\right|^{2}+|\nabla v|^{2}+\left(\gamma+\frac{\alpha\left|\gamma^{\prime}\right|}{\rho+2}\right)|u|^{\rho+2}\right) \aleph_{m}^{2} d x d t .
\end{aligned}
$$

So it can be estimated by

$$
\begin{aligned}
& c_{0} \int_{\left(S_{m+1}^{t} \backslash S_{m}^{t}\right) \times \sigma}\left|v^{\prime}\right|^{2}+|v|^{2}+|\nabla v|^{2}+\gamma|u|^{\rho+2} d x d t \\
& \quad+\int_{Q_{t}} 2 \gamma\left(|u|^{\rho} u\right) u_{\infty} \aleph_{m}^{2} d x d t+\int_{Q_{t}} 2 w \aleph_{m}^{2} F+2 \alpha \nu^{\prime} \aleph_{m}^{2} F d x d t .
\end{aligned}
$$


Here and in the sequel, we notice that

$$
\left(|u|^{\rho} u\right) u_{\infty} \leq \frac{(\rho+1) \varepsilon}{\rho+2}|u|^{\rho+2}+\frac{1}{(\rho+2) \varepsilon^{(\rho+1)}}\left|u_{\infty}\right|^{\rho+2} .
$$

The same inequality, for $p=q=2$, yields that

$$
\begin{aligned}
& 2 w \aleph_{m}^{2} F+2 \alpha v^{\prime} F \leq \varepsilon\left(v^{2}+\left|v^{\prime}\right|^{2}\right)+\frac{1+\alpha^{2}}{\varepsilon} F^{2}, \\
& 2 v v^{\prime} \leq v^{2}+\left|v^{\prime}\right|^{2}, \\
& 2 v|\nabla v| \leq v^{2}+|\nabla v|^{2} .
\end{aligned}
$$

So

$$
\begin{aligned}
& c_{0} \int_{\left(S_{m+1}^{t} \mid S_{m}^{t}\right) \times \sigma}\left|v^{\prime}\right|^{2}+|v|^{2}+|\nabla v|^{2}+\gamma|u|^{\rho+2} d x d t \\
& \quad+c_{1} \varepsilon \int_{Q_{t}}\left(\left|v^{\prime}\right|^{2}+\gamma|u|^{\rho+2}\right) \aleph_{m}^{2} d x d t+\frac{c_{1}}{\varepsilon^{(\rho+1)}} \int_{Q t}\left(F^{2}+\gamma\left|u_{\infty}\right|^{\rho+2}\right) \aleph_{m}^{2} d x d t
\end{aligned}
$$

$=0$.

Since $\sigma$ is bounded, Poincarés inequality yields that

$$
\int_{\Omega_{t}}|v(t)|^{2} \aleph_{m}^{2}(t) d x \leq c_{\sigma}^{2} \int_{\Omega_{t}}\left|\nabla_{X_{2}} v(t)\right|^{2} \aleph_{m}^{2}(t) d x \leq c_{\sigma}^{2} \int_{\Omega_{t}}|\nabla v(t)|^{2} \aleph_{m}^{2}(t) d x
$$

where $c_{\sigma}$ is a positive Poincaré constant.

By applying Poincarés inequality again, we have

$$
\begin{aligned}
& c_{2} \int_{\left(S_{m+1}^{t} \mid S_{m}^{t}\right) \times \sigma}\left|v^{\prime}\right|^{2}+|\nabla v|^{2}+\gamma|u|^{\rho+2} d x d t \\
& \quad+c_{2} \varepsilon \int_{Q_{t}}\left(\left|v^{\prime}\right|^{2}+|\nabla v|^{2}+\gamma|u|^{\rho+2}\right) \aleph_{m}^{2} d x d t+\frac{c_{2}}{\varepsilon^{(\rho+1)}} \int_{Q_{t}}\left(F^{2}+\gamma\left|u_{\infty}\right|^{\rho+2}\right) \aleph_{m}^{2} d x d t .
\end{aligned}
$$

Thus

$$
\begin{aligned}
& \int_{\Omega_{t}}\left(\delta_{0}\left|v^{\prime}(t)\right|^{2}+\alpha|\nabla v(t)|^{2}+\frac{2 \alpha \gamma(t)}{\rho+2}|u(t)|^{\rho+2}\right) \aleph_{m}^{2}(t) d x \\
& \quad+2 \int_{Q t}\left(\iota \delta_{0}\left|v^{\prime}\right|^{2}+|\nabla v|^{2}+\left(\gamma+\frac{\alpha\left|\gamma^{\prime}\right|}{\rho+2}\right)|u|^{\rho+2}\right) \aleph_{m}^{2} d x d t \\
& \leq c_{2} \int_{\left(S_{m+1}^{t} \mid S_{m}^{t}\right) \times \sigma}\left|v^{\prime}\right|^{2}+|\nabla v|^{2}+\gamma|u|^{\rho+2} d x d t \\
& \quad+c_{2} \varepsilon \int_{Q t}\left(\left|v^{\prime}\right|^{2}+|\nabla v|^{2}+\gamma|u|^{\rho+2}\right) \aleph_{m}^{2} d x d t \\
& \quad+\frac{c_{2}}{\varepsilon^{(\rho+1)}} \int_{Q_{t}}\left(F^{2}+\gamma\left|u_{\infty}\right|^{\rho+2}\right) \aleph_{m}^{2} d x d t .
\end{aligned}
$$


Considering that $\varepsilon$ is small enough, we have

$$
\begin{aligned}
& \int_{\Omega_{t}}\left(\left|v^{\prime}(t)\right|^{2}+|\nabla v(t)|^{2}+\gamma(t)|u(t)|^{\rho+2}\right) \aleph_{m}^{2}(t) d x \\
& \quad+\int_{Q_{t}}\left(\left|v^{\prime}\right|^{2}+|\nabla v|^{2}+\gamma|u|^{\rho+2}\right) \aleph_{m}^{2} d x d t \\
& \leq c_{3} \int_{\left(S_{m+1}^{t} \mid S_{m}^{t}\right) \times \sigma}\left|v^{\prime}\right|^{2}+|\nabla v|^{2}+\gamma|u|^{\rho+2} d x d t \\
& \quad+c_{3} \int_{Q_{t}}\left(F^{2}+\gamma\left|u_{\infty}\right|^{\rho+2}\right) \aleph_{m}^{2} d x d t \\
& <+\infty
\end{aligned}
$$

This completes the proof.

\section{Conclusions}

In this paper, we generalized the Poisson-Sch type inequalities by using new identities involving new Green-Sch's functions. As applications in quantum calculus, we estimated the size of weighted Schrödingerean harmonic Bergman functions and $L^{p}$-norm size of partial derivatives of extended Poisson-Sch kernel functions associated with the Schrödinger operator in the upper half space.

\footnotetext{
Acknowledgements

We would like to thank the editor, the associate editor, and the anonymous referees for their careful reading and constructive comments which have helped us to significantly improve the presentation of the paper.

\section{Funding}

This work was supported by the National Natural Science Foundation of China (No. 61403356) and the Zhejiang Provincial Natural Science Foundation of China (No. LY18F030012).
}

Availability of data and materials

Not applicable.

Competing interests

The authors declare that they have no competing interests.

\section{Authors' contributions}

All authors read and approved the final manuscript.

\section{Author details}

${ }^{1}$ School of Mathematics and Computer Science, Panzhihua University, Panzhihua, China. ${ }^{2}$ School of Mechanotronics and Vehicle Engineering, Qingyuan Polytechnic, Qingyuan, China. ${ }^{3}$ Shenzhen Quantum Wisdom Culture Development Company, Shenzhen, China. ${ }^{4}$ Centre for Quantum Technologies, National University of Singapore, Singapore, Singapore.

\section{Publisher's Note}

Springer Nature remains neutral with regard to jurisdictional claims in published maps and institutional affiliations.

Received: 21 April 2018 Accepted: 8 June 2018 Published online: 03 July 2018

\section{References}

1. Abramowitz, M., Stegun, I.A.: Handbook of Mathematical Function, 10th edn. National Bureau of Standards, Washington (1972)

2. Axler, S., Bourdon, P., Ramey, W.: Harmonic Function Theory. Springer, New York (1992)

3. Beatrous, F.: Behavior of holomorphic functions near weakly pseudoconvex boundary points. Indiana Univ. Math. J. 40(40), 915-966 (1991)

4. Bohner, M., Peterson, A.: Dynamic Equations on Time Scales. Birkhäuser, Boston (2001)

5. Bresters, D.W.: On the equation of Euler-Poisson-Darboux. SIAM J. Math. Anal. 1(12), 31-41 (1973)

6. Coifman, R., Rochberg, R.: Representation theorems for holomorphic and harmonic functions in $L^{p}$. Astérisque 77(13), $11-66(1980)$ 
7. Cottle, R.W.: Nonlinear programs with positively bounded Jacobians. Ph.D. dissertation, Department of Mathematics, University of California, Berkeley (1964)

8. Fulton, C.T.: Two-point boundary value problems with eigenparameter contained in the boundary conditions. Proc R. Soc. Edinb. 77A, 293-308 (1977)

9. Gasiorowicz, S.: Elementary Particle Physics. Wiley, New York (1966)

10. Gelfand, I., Shilov, G.: Generalized Functions, vol. 1. Academic Press, New York (1964)

11. Glowinski, R., Lions, L.J., Trémolières, R.: Numerical Analysis of Variational Inequalities. North-Holland, Amsterdam (1981)

12. Hartman, P., Stampacchia, G.: On some nonlinear elliptic differential functional equations. Acta Math. 115, 153-188 (1966)

13. Hedenmalm, H., Korenblum, B., Zhu, K.: Theory of Bergman Spaces. Springer, New York (2000)

14. Huang, J.: Solution to the inverse problem of the 1-D wave equation. J. Math. Phys. 30(3), 735-739 (1989)

15. Ikegami, T.: Compactifications of Martin type of harmonic spaces. Osaka J. Math. 23(3), 653-680 (1986)

16. Lions, L.J., Stampaccia, G.: Variational inequalities. Commun. Pure Appl. Math. 20, 493-512 (1967)

17. Panagiotopoulos, P.D.: Inequality Problems in Mechanics and Applications. Birkhäuser, Boston (1985)

18. Parida, J., Sen, A.: A variational-like inequality problems for multi-functions with applications. J. Math. Anal. Appl. 124, 73-81 (1987)

19. Passare, M.: Residues, currents, and their relation to ideals of holomorphic functions. Math. Scand. 62(1), 75-152 (1988)

20. Qiao, Y., Zhou, Z.: Positive solutions for a class of Hadamard fractional differential equations on the infinite interval. Math. Appl. 30(3), 589-594 (2017)

21. Shu, C., Chen, L., Vargas-De-Teón, R.: New Poisson type inequalities with respect to a cone and their applications. J. Inequal. Appl. 2017, Article ID 114 (2017). https://doi.org/10.1186/s13660-017-1387-y

22. Wang, J., Pu, J., Zama, A.: Solutions of the Dirichlet-Schrödinger problems with continuous data admitting arbitrary growth property in the boundary. Adv. Difference Equ. 2016, Article ID 33 (2016). https://doi.org/10.1186/s13662-016-0751-4

23. Zhang, G., Zhao, T.: Nonconvex retracts constructed by quasiconvex functionals. J. Northeast. Univ. Nat. Sci. 34(7), 1061-1064 (2013)

24. Zhu, K.: Operator Theory in Function Spaces. Dekker, New York (1990)

\section{Submit your manuscript to a SpringerOpen ${ }^{\circ}$ journal and benefit from:}

- Convenient online submission

- Rigorous peer review

- Open access: articles freely available online

- High visibility within the field

- Retaining the copyright to your article

Submit your next manuscript at $\gg$ springeropen.com 